\title{
OPTIFLOWTM(OF) VERSUS VAPOTHERM®(VT) AS EXTENDED WEANING MODE FROM NASAL CONTINUOUS AIRWAY PRESSURE (NCPAP) IN PRETERM INFANTS $\leq 28$ WEEKS GESTATIONAL AGE (GA)
}

L. Mahoney, J.R. Fernandez Alvarez, R. Gandhi, R. Bomont, C. Garland, H. Rabe

Department of Neonatology, Brighton and Sussex University Hospitals NHS Trust, Brighton, UK

Background: Current evidence supports continuous over discontinuous NCPAP weaning. The best strategy remains unknown. Humidified high flow nasal cannula (HHFNC) reduces NCPAP time in infants $<28 \mathrm{GA}$.

Aim: To compare the two most frequently used HHFNC devices in weaning from NCPAP.

Methods: Matched-pair case-control study in infants $\leq 28 \mathrm{GA}$ born in a single tertiary neonatal centre between 2006-2010. Patients were matched for GA, birthweight (BW), antenatal steroids (AS), surfactant, mechanical ventilation and NCPAP. Outcome measures were duration of non-invasive respiratory support (HHFNC support and nasal cannula oxygen), oxygen requirement at 36GA, postnatal steroids, pneumothorax, length of stay (LOS) and discharge weight (DW). Results reported as median $\left(25^{\text {th }}-75^{\text {th }}\right.$ quartile). Statistics: Wilcoxon-and McNemar-test, $\mathrm{p}<0.05$.

Results: 18 (VT-group) and 14 (OF-group) patients with a GA and BW of 28GA(27-28) and 1170g(1031$1252)$ and $27 \mathrm{GA}(26-28)$ and $1051 \mathrm{~g}(928-1230)$ were recruited respectively. In the VT-group 15/18 received AS and 10/18 surfactant versus 10/14 and 10/14 in the OF-group. 10/18 of patients in the VT-group were ventilated for 1 day $(0-3)$ and $16 / 18$ on NCPAP for 5 days(2-7) as compared to $10 / 14$ for 2 days $(0-13)$ and $12 / 14$ for 4days(2-9) in the OF-group. There was no statistically significant difference in any outcome measure compared: Patients spent 7 days(3-18) on VT versus 7 days(0-23) on OF without pneumothorax. 2/18 and 3/14 required oxygen at 36GA. Only one in the VF group required dexamethasone. LOS and DW were 62days(54-74) and 2168g(2009-2383) versus 67days(53-99) and 2221g(2078-2703).

Conclusion: Optiflow ${ }^{\mathrm{TM}}$ and Vapotherm ${ }^{\circledR}$ seem to be equally effective for weaning from NCPAP without increasing the risk of pneumothorax or bronchopulmonary dysplasia. 